\title{
Some Notes on Matrix Transforms of Summability Domains of Cesàro Matrices
}

\author{
A. Aasma \\ Tallinn University of Technology, Department of Economics \\ Akadeemia tee 3, 12618, Tallinn, Estonia \\ E-mail: ants.aasma@tseba.ttu.ee
}

Received September 25, 2009; revised December 18, 2009; published online April 20, 2010

\begin{abstract}
In this paper sufficient conditions for a matrix $M=\left(m_{n k}\right)\left(m_{n k}\right.$ are Cesàro numbers $A_{n-k}^{s}, s \in \mathcal{C}$ if $k \leq n$ and $m_{n k}=0$ if $k>n$ ) to be a transform from the summability domain of the Cesàro method $C^{\alpha}$ into the summability domain of another Cesàro method $C^{\beta}$, where $\alpha, \beta \in \mathcal{C} \backslash\{-1,-2, \ldots\}$, are found.
\end{abstract}

Keywords: matrix transformations, summability method of Cesàro.

AMS Subject Classification: 40C05; 40G05.

\section{Introduction}

Let $C^{\alpha}=\left(c_{n k}\right), \alpha \in \mathcal{C} \backslash\{-1,-2, \ldots\}$, be a series-to-sequence Cesàro method, i.e. (see [4] or [5])

$$
c_{n k}= \begin{cases}\frac{A_{n-k}^{\alpha}}{A_{n}^{\alpha}} & (k \leq n), \\ 0 & (k>n),\end{cases}
$$

where $A_{n}^{\alpha}=\left(\begin{array}{c}n+\alpha \\ n\end{array}\right)$ are Cesàro numbers. Throughout this paper we assume that summation indices run from 0 to $\infty$ unless otherwise specified. A series $x:=$ $\sum x_{k}$ is said to be $C^{\alpha}$-summable if the sequence $C^{\alpha} x=\left(C_{n}^{\alpha} x\right)$ is convergent, where

$$
C_{n}^{\alpha} x=\sum_{k=0}^{n} c_{n k} x_{k} .
$$

We denote the domain of all $C^{\alpha}$-summable series by $c_{C^{\alpha}}$, i.e.

$$
c_{C^{\alpha}}:=\left\{x=\left(x_{n}\right) \mid \lim _{n \rightarrow \infty} C_{n}^{\alpha} x \text { exists }\right\} .
$$

In $[1,3,10]$ necessary and sufficient conditions for a matrix $M$ with real or complex entries to be a transform from $c_{C^{\alpha}}$ into $c_{C^{\beta}}$ for $\alpha, \beta \in \mathcal{R}$ or $\alpha, \beta \in$ $\mathcal{C} \backslash\{-1,-2, \ldots\}$ are described. The summability domains (and the subsets of 
summability domains) of different Cesàro methods are compared in several papers (see, for example, $[2,7,8]$ ). For double Cesàro methods this problem has in recent years been considered, for example, in $[6,9]$.

In the present paper the particular subcase of the above-described problem is studied: sufficient conditions for a matrix $M=\left(m_{n k}\right)$, defined by the relation

$$
m_{n k}= \begin{cases}A_{n-k}^{s} & (k \leq n, s \in \mathcal{C}), \\ 0 & (k>n)\end{cases}
$$

to be a transform from $c_{C^{\alpha}}$ into $c_{C^{\beta}}, \alpha, \beta \in \mathcal{C} \backslash\{-1,-2, \ldots\}$ are found. It is easy to see that this problem is equivalent to the problem of finding sufficient conditions for $c_{C^{\alpha}} \subset c_{G}$, where $G:=C^{\beta} M$.

\section{Auxiliary Results}

For the proof of main results we need the following properties of Cesàro numbers (see [4], p. 77-81):

$$
\begin{aligned}
& \sum_{k=0}^{n} A_{n-k}^{\alpha} A_{k}^{\beta}=A_{n}^{\alpha+\beta+1} \text { for every } \alpha, \beta \in \mathcal{C}, \\
& \left|A_{n}^{\alpha}\right| \leq K_{1}(n+1)^{R e \alpha} \quad \text { for every } \alpha \in \mathcal{C}, K_{1}>0, \\
& \left|A_{n}^{\alpha}\right| \geq K_{2}(n+1)^{\text {Re } \alpha} \quad \text { for } \alpha \in \mathcal{C} \backslash\{-1,-2, \ldots\}, K_{2}>0 .
\end{aligned}
$$

Further we also use the following lemma.

Lemma 1. Let $\alpha \in \mathcal{C}, \beta \in \mathcal{C}$. The following assertions hold:

(A) If $\operatorname{Re} \alpha \neq-1$ and $\operatorname{Re} \beta \neq-1$, or $\alpha=\beta=-1$, then

$$
D_{n}:=\sum_{k=0}^{n}\left|A_{n-k}^{\alpha} A_{k}^{\beta}\right|=\mathcal{O}\left[(n+1)^{R e \alpha}\right]+\mathcal{O}\left[(n+1)^{R e \beta}\right]+\mathcal{O}\left[(n+1)^{\operatorname{Re}(\alpha+\beta)+1}\right] .
$$

(B) If $\operatorname{Re} \beta=-1$, then

$$
D_{n}= \begin{cases}\mathcal{O}\left[(n+1)^{\operatorname{Re} \alpha} \ln (n+1)\right] & (\operatorname{Re} \alpha \geq-1), \\ \mathcal{O}\left[(n+1)^{-1}\right] & (\operatorname{Re} \alpha<-1) .\end{cases}
$$

(C) If $\operatorname{Re} \alpha=-1$, then

$$
D_{n}= \begin{cases}\mathcal{O}\left[(n+1)^{\operatorname{Re} \beta} \ln (n+1)\right] & (\operatorname{Re} \beta \geq-1) \\ \mathcal{O}\left[(n+1)^{-1}\right] & (\operatorname{Re} \beta<-1)\end{cases}
$$

Proof. First we note that for all $\alpha, \beta \in \mathcal{R}$ relation (2.4) is proved, for example, in [4], p. 79-81. Let now $\operatorname{Re} \alpha \neq-1, \operatorname{Re} \beta \neq-1$. Then by (2.2) and (2.3) there 
exist $K_{1}^{1}, K_{1}^{2}, K_{2}^{1}, K_{2}^{2}>0$ so that

$$
D_{n} \leq K_{1}^{1} K_{1}^{2} \sum_{k=0}^{n}(n-k+1)^{R e \alpha}(k+1)^{R e \beta} \leq K_{1}^{1} K_{1}^{2} K_{2}^{1} K_{2}^{2} \sum_{k=0}^{n}\left|A_{n-k}^{R e \alpha} A_{k}^{R e \beta}\right| .
$$

Hence relation (2.4) holds, since $\operatorname{Re} \alpha, \operatorname{Re} \beta \in \mathcal{R}$. Thus assertion (A) is satisfied.

Let now $\operatorname{Re} \beta=-1$. Then with the help of relation $(2.2)$ we have

$$
D_{n} \leq M V_{n}, \quad M>0
$$

where

$$
V_{n}:=\sum_{k=0}^{n} v_{n k}, \quad v_{n k}:=(n-k+1)^{\operatorname{Re} \alpha}(k+1)^{-1} .
$$

Further we can write

$$
V_{n}= \begin{cases}\sum_{k=0}^{\frac{n}{2}-1} v_{n k}+\left(\frac{n}{2}+1\right)^{R e \alpha-1}+\sum_{k=\frac{n}{2}+1}^{n} v_{n k} & (n \text { is even }), \\ \sum_{k=0}^{\frac{n-1}{2}} v_{n k}+\sum_{k=\frac{n+1}{2}}^{n} v_{n k} & (n \text { is odd }),\end{cases}
$$

i.e.,

$$
\begin{aligned}
V_{n}= & (n+1)^{\operatorname{Re} \alpha} 1^{-1}+n^{\operatorname{Re} \alpha} 2^{-1}+\cdots+\left(\frac{n}{2}+3\right)^{\operatorname{Re} \alpha}\left(\frac{n}{2}-1\right)^{-1} \\
& +\left(\frac{n}{2}+2\right)^{\operatorname{Re} \alpha}\left(\frac{n}{2}\right)^{-1}+\left(\frac{n}{2}+1\right)^{\operatorname{Re} \alpha-1}+\left(\frac{n}{2}\right)^{\operatorname{Re} \alpha}\left(\frac{n}{2}+2\right)^{-1} \\
& +\cdots+2^{\operatorname{Re} \alpha} n^{-1}+1^{\operatorname{Re} \alpha}(n+1)^{-1}
\end{aligned}
$$

for an even number $n$, and

$$
\begin{aligned}
V_{n}= & (n+1)^{\operatorname{Re} \alpha} 1^{-1}+n^{\operatorname{Re} \alpha} 2^{-1}+\cdots+\left(\frac{n+5}{2}\right)^{\operatorname{Re} \alpha}\left(\frac{n-1}{2}\right)^{-1} \\
& +\left(\frac{n+3}{2}\right)^{\operatorname{Re} \alpha}\left(\frac{n+1}{2}\right)^{-1}+\left(\frac{n+1}{2}\right)^{\operatorname{Re} \alpha}\left(\frac{n+3}{2}\right)^{-1} \\
& +\left(\frac{n-1}{2}\right)^{\operatorname{Re} \alpha}\left(\frac{n+1}{2}\right)^{-1}+\cdots+2^{\operatorname{Re} \alpha} n^{-1}+1^{\operatorname{Re} \alpha}(n+1)^{-1}
\end{aligned}
$$

for an odd number $n$. Let us suppose first that $\operatorname{Re} \alpha \geq-1$. Then

$$
\begin{aligned}
& (n+1)^{\operatorname{Re} \alpha} 1^{-1} \geq 1^{\operatorname{Re} \alpha}(n+1)^{-1}, \\
& n^{\operatorname{Re} \alpha} 2^{-1} \geq 2^{\operatorname{Re} \alpha} n^{-1}, \\
& \ldots \ldots \ldots \ldots \ldots \ldots \ldots \ldots \ldots \ldots \ldots \ldots \ldots \ldots \ldots \ldots \ldots \ldots \ldots \ldots \\
& \left(\frac{n}{2}+2\right)^{\operatorname{Re} \alpha}\left(\frac{n}{2}\right)^{-1} \geq\left(\frac{n}{2}\right)^{\operatorname{Re} \alpha}\left(\frac{n}{2}+2\right)^{-1},
\end{aligned}
$$


if $n$ is an even number, and

$$
\begin{aligned}
& (n+1)^{\operatorname{Re} \alpha} 1^{-1} \geq 1^{\operatorname{Re} \alpha}(n+1)^{-1}, \\
& n^{\operatorname{Re} \alpha} 2^{-1} \geq 2^{\operatorname{Re} \alpha} n^{-1}, \\
& \ldots \ldots \ldots \ldots \ldots \ldots \ldots \ldots \ldots \ldots \ldots \ldots \ldots \ldots \ldots \ldots \ldots \ldots \\
& \left(\frac{n+3}{2}\right)^{\operatorname{Re} \alpha}\left(\frac{n+1}{2}\right)^{-1} \geq\left(\frac{n+1}{2}\right)^{\operatorname{Re} \alpha}\left(\frac{n+3}{2}\right)^{-1},
\end{aligned}
$$

if $n$ is an odd number. Consequently

$$
V_{n} \leq \begin{cases}2 \sum_{k=0}^{\frac{n}{2}-1}(n-k+1)^{\operatorname{Re} \alpha}(k+1)^{-1}+\left(\frac{n}{2}+1\right)^{\operatorname{Re} \alpha-1} & (n \text { is even }), \\ 2 \sum_{k=0}^{\frac{n-1}{2}}(n-k+1)^{\operatorname{Re} \alpha}(k+1)^{-1} & (n \text { is odd }) .\end{cases}
$$

Therefore

$$
V_{n} \leq \begin{cases}2\left(\frac{n}{2}+2\right)^{\operatorname{Re} \alpha} \sum_{k=0}^{\frac{n}{2}-1}(k+1)^{-1}+\left(\frac{n}{2}+1\right)^{\operatorname{Re} \alpha-1} & (n \text { is even }), \\ 2\left(\frac{n+3}{2}\right)^{\operatorname{Re} \alpha} \sum_{k=0}^{\frac{n-1}{2}}(k+1)^{-1} & (n \text { is odd })\end{cases}
$$

if $-1 \leq \operatorname{Re} \alpha \leq 0$, and

$$
V_{n} \leq \begin{cases}2(n+1)^{\operatorname{Re} \alpha} \sum_{k=0}^{\frac{n}{2}-1}(k+1)^{-1}+\left(\frac{n}{2}+1\right)^{\operatorname{Re} \alpha-1} & (n \text { is even }) \\ 2(n+1)^{\operatorname{Re} \alpha} \sum_{k=0}^{\frac{n-1}{2}}(k+1)^{-1} & (n \text { is odd })\end{cases}
$$

if $\operatorname{Re} \alpha>0$. Hence

$$
D_{n}=\mathcal{O}\left[(n+1)^{R e \alpha} \ln (n+1)\right] ; \operatorname{Re} \alpha \geq-1 .
$$

We assume now that $\operatorname{Re} \alpha<-1$. Then

$$
\begin{aligned}
& (n+1)^{\text {Re } \alpha} 1^{-1} \leq 1^{\text {Re } \alpha}(n+1)^{-1} \\
& n^{\text {Re } \alpha} 2^{-1} \leq 2^{\text {Re } \alpha} n^{-1}, \\
& \ldots \ldots \ldots \ldots \ldots \ldots \ldots \ldots \ldots \ldots \ldots \ldots \ldots \ldots \ldots \ldots \ldots \ldots \ldots \ldots \\
& \left(\frac{n}{2}+2\right)^{\operatorname{Re} \alpha}\left(\frac{n}{2}\right)^{-1} \leq\left(\frac{n}{2}\right)^{\operatorname{Re} \alpha}\left(\frac{n}{2}+2\right)^{-1},
\end{aligned}
$$

if $n$ is an even number, and

$$
\begin{aligned}
& (n+1)^{\operatorname{Re} \alpha} 1^{-1} \leq 1^{\operatorname{Re} \alpha}(n+1)^{-1} \\
& n^{\operatorname{Re} \alpha} 2^{-1} \leq 2^{\operatorname{Re} \alpha} n^{-1} \\
& \ldots \ldots \ldots \ldots \ldots \ldots \ldots \ldots \ldots \ldots \ldots \ldots \ldots \ldots \ldots \ldots \ldots \ldots \ldots \\
& \left(\frac{n+3}{2}\right)^{\operatorname{Re} \alpha}\left(\frac{n+1}{2}\right)^{-1} \leq\left(\frac{n+1}{2}\right)^{\operatorname{Re} \alpha}\left(\frac{n+3}{2}\right)^{-1},
\end{aligned}
$$


if $n$ is an odd number. Consequently

$$
V_{n} \leq \begin{cases}2 \sum_{k=\frac{n}{2}+1}^{n}(n-k+1)^{\operatorname{Re} \alpha}(k+1)^{-1}+\left(\frac{n}{2}+1\right)^{\operatorname{Re} \alpha-1} & (n \text { is even }), \\ 2 \sum_{k=\frac{n+1}{2}}^{n}(n-k+1)^{\operatorname{Re} \alpha}(k+1)^{-1} & (n \text { is odd }) .\end{cases}
$$

Therefore

$$
V_{n} \leq \begin{cases}4(n+4)^{-1} \sum_{k=\frac{n}{2}+1}^{n}(n-k+1)^{\text {Re } \alpha}+\left(\frac{n}{2}+1\right)^{\text {Re } \alpha-1} & (n \text { is even }), \\ 4(n+3)^{-1} \sum_{k=\frac{n+1}{2}}^{n}(n-k+1)^{\text {Re } \alpha} & (n \text { is odd })\end{cases}
$$

Hence

$$
\left.D_{n}=\mathcal{O}\left[(n+1)^{-1}\right] ; \operatorname{Re} \alpha<-1\right) .
$$

Consequently assertion (B) holds by (2.5) and (2.6). The proof of assertion (C) is similar to the proof of assertion (B). So we omit it.

\section{Main Results}

Now we are able to prove the main result of this paper.

Theorem 1. Let $\alpha, \beta \in \mathcal{C} \backslash\{-1,-2, \ldots\}$ and $s \in \mathcal{C}$. If Re $s<-1$ and Re $s<$ $\operatorname{Re} \alpha \leq \operatorname{Re} \beta$, then the matrix $M=\left(m_{n k}\right)$, defined by relation (1.1), transforms $c_{C^{\alpha}}$ into $c_{C^{\beta}}$.

Proof. It is sufficient to show that $c_{C^{\alpha}} \subset c_{G}$, where $G=C^{\beta} M:=\left(g_{n k}\right)$. Using equality (2.1), we get

$$
g_{n l}=\frac{1}{A_{n}^{\beta}} \sum_{k=l}^{n} A_{n-k}^{\beta} A_{k-l}^{s}=\frac{1}{A_{n}^{\beta}} \sum_{k=0}^{n-l} A_{n-l-k}^{\beta} A_{k}^{s}=\frac{A_{n-l}^{\beta+s+1}}{A_{n}^{\beta}} .
$$

As the inverse matrix $\left(\eta_{l k}\right)$ of $C^{\alpha}=\left(c_{n k}\right)$ is defined by the equalities (see [4], p. 86)

$$
\eta_{l k}= \begin{cases}A_{k}^{\alpha} A_{l-k}^{-\alpha-2} & (k \leq l) \\ 0 & (k>l)\end{cases}
$$

for every $x=\left(x_{k}\right) \in c_{C^{\alpha}}$ we get

$$
\sum_{k=0}^{n} g_{n k} x_{k}=\sum_{k=0}^{n} \gamma_{n k} y_{k}
$$

where $y_{k}:=C_{k}^{\alpha} x$ and $\gamma_{n k}=A_{n-k}^{\beta+s-\alpha} A_{k}^{\alpha} / A_{n}^{\beta}$ by equality (2.1). Consequently, for $c_{C^{\alpha}} \subset c_{G}$ it is sufficient to show by the well-known theorem of Kojima-Schur that

$$
\text { there exists the finite limits } \lim _{n} \gamma_{n k}, \lim _{n} \sum_{k=0}^{n} \gamma_{n k} \text {, }
$$


and

$$
\sum_{k}\left|\gamma_{n k}\right|=\mathcal{O}(1)
$$

since the sequence $\left(y_{k}\right)$ is convergent for every $x \in c_{C^{\alpha}}$. Thus, with the help of relations (2.1)-(2.3) we have

$$
\left|\sum_{k=0}^{n} \gamma_{n k}\right|=\left|\frac{A_{n}^{\beta+s+1}}{A_{n}^{\beta}}\right|=\mathcal{O}(1)(n+1)^{R e s+1}=o(1)
$$

(since $\operatorname{Re} s+1<0$ ), and

$$
\begin{aligned}
\left|\gamma_{n k}\right| & =\mathcal{O}(1) \frac{(n-k+1)^{\operatorname{Re}(\beta+s-\alpha)}}{(n+1)^{\operatorname{Re} \beta}} \\
& =\mathcal{O}(1)\left(1-\frac{k}{n+1}\right)^{\operatorname{Re} \beta}(n-k+1)^{\operatorname{Re}(s-\alpha)}=o(1)
\end{aligned}
$$

(since $\operatorname{Re}(s-\alpha)<0)$. Thus condition (3.1) is fulfilled.

The proof of validity of condition (3.2) we divide into three parts.

1) Let $\operatorname{Re}(\beta+s-\alpha) \neq-1, \operatorname{Re} \alpha \neq-1$, or $\beta+s-\alpha=\alpha=-1$. Then we get

$$
\begin{aligned}
S_{n}:=\sum_{k=0}^{n}\left|A_{n-k}^{\beta+s-\alpha} A_{k}^{\alpha}\right|=\mathcal{O}\left[(n+1)^{\operatorname{Re}(\beta+s-\alpha)}\right] & +\mathcal{O}\left[(n+1)^{\operatorname{Re} \alpha}\right] \\
& +\mathcal{O}\left[(n+1)^{\operatorname{Re}(\beta+s)+1}\right]
\end{aligned}
$$

by Lemma 1 . If

$$
L:=\max \{\operatorname{Re}(\beta+s-\alpha), \operatorname{Re} \alpha, \operatorname{Re}(\beta+s)+1\}=\operatorname{Re}(\beta+s-\alpha),
$$

then $S_{n}=\mathcal{O}\left[(n+1)^{R e(\beta+s-\alpha)}\right]$, and consequently with the help of $(2.3)$ we have

$$
T_{n}:=\sum_{k=0}^{n}\left|\gamma_{n k}\right|=\frac{S_{n}}{\left|A_{n}^{\beta}\right|}=\mathcal{O}\left[(n+1)^{R e(s-\alpha)}\right]=\mathcal{O}(1) .
$$

If $L=\operatorname{Re}(\beta+s)+1$, then using (2.3) we can conclude that

$$
S_{n}=\mathcal{O}\left[(n+1)^{\operatorname{Re}(\beta+s)+1}\right]
$$

and therefore

$$
T_{n}=\mathcal{O}\left[(n+1)^{R e s+1}\right]=\mathcal{O}(1) .
$$

If $L=\operatorname{Re} \alpha$, then $S_{n}=\mathcal{O}\left[(n+1)^{R e} \alpha\right]$, and hence

$$
T_{n}=\mathcal{O}\left[(n+1)^{\operatorname{Re}(\alpha-\beta)}\right]=\mathcal{O}(1),
$$

i.e. condition (3.2) holds. 
2) Let $\operatorname{Re} \alpha=-1$. Then

$$
S_{n}= \begin{cases}\mathcal{O}\left[(n+1)^{\operatorname{Re}(\beta+s-\alpha)} \ln (n+1)\right] & (\operatorname{Re}(\beta+s-\alpha) \geq-1), \\ \mathcal{O}\left[(n+1)^{-1}\right] & (\operatorname{Re}(\beta+s-\alpha)<-1),\end{cases}
$$

and consequently

$$
T_{n}= \begin{cases}\mathcal{O}\left[(n+1)^{\operatorname{Re}(s-\alpha)} \ln (n+1)\right] & (\operatorname{Re}(\beta+s-\alpha) \geq-1), \\ \mathcal{O}\left[(n+1)^{-\operatorname{Re} \beta-1}\right] & (\operatorname{Re}(\beta+s-\alpha)<-1) .\end{cases}
$$

Therefore $T_{n}=\mathcal{O}(1)$, because $\operatorname{Re}(s-\alpha)<0$ and $\operatorname{Re} \beta \geq \operatorname{Re} \alpha=-1$, i.e. condition (3.2) holds.

3) Let $\operatorname{Re}(\beta+s-\alpha)=-1$. Then

$$
S_{n}= \begin{cases}\mathcal{O}\left[(n+1)^{\operatorname{Re} \alpha} \ln (n+1)\right] & (\operatorname{Re} \alpha \geq-1), \\ \mathcal{O}\left[(n+1)^{-1}\right] & (\operatorname{Re} \alpha<-1),\end{cases}
$$

and consequently

$$
T_{n}= \begin{cases}\mathcal{O}\left[(n+1)^{\operatorname{Re}(\alpha-\beta)} \ln (n+1)\right] & (\operatorname{Re} \alpha \geq-1) \\ \mathcal{O}\left[(n+1)^{-\operatorname{Re} \beta-1}\right] & (\operatorname{Re} \alpha<-1) .\end{cases}
$$

Therefore $T_{n}=\mathcal{O}(1)$, because $\operatorname{Re}(\alpha-\beta)=\operatorname{Re} s+1<0$ and $-\operatorname{Re} \beta-1=$ $\operatorname{Re}(s-\alpha)<0$, i.e. condition (3.2) holds.

It is well known that $C^{\beta}$ includes $C^{\alpha}$, i.e. $c_{C^{\beta}} \supseteq c_{C^{\alpha}}$, if $\operatorname{Re} \beta>\operatorname{Re} \alpha>-1$ (see [4], p. 87). Therefore for real numbers $\alpha, \beta$ we get

Corollary 1. Let $\alpha, \beta \in \mathcal{R}, s \in \mathcal{C}$ with $\alpha, \beta>-1$, Re $s<-1$ and $M$ be defined by (1.1). If $M$ transforms $c_{C^{\alpha}}$ into $c_{C^{\beta}}$, then $C^{\beta}$ includes $C^{\alpha}$.

Proof. We see from the proof of Theorem 1 that the validity of condition (3.2) is necessary for $M$ to be a transformation from $c_{C^{\alpha}}$ into $c_{C^{\beta}}$. We prove that condition (3.2) is not satisfied for $\beta<\alpha$. Indeed, by (2.2) and (2.3) there exists a number $K>0$ so that

$$
T_{n}=\sum_{k=0}^{n}\left|\frac{A_{n-k}^{\beta+s-\alpha} A_{k}^{\alpha}}{A_{n}^{\beta}}\right|=\sum_{k=0}^{n-1}\left|\frac{A_{n-k}^{\beta+s-\alpha} A_{k}^{\alpha}}{A_{n}^{\beta}}\right|+\left|\frac{A_{n}^{\alpha}}{A_{n}^{\beta}}\right| \geq\left|\frac{A_{n}^{\alpha}}{A_{n}^{\beta}}\right| \geq K\left[(n+1)^{\operatorname{Re}(\alpha-\beta)}\right] .
$$

As the sequence $\left((n+1)^{R e(\alpha-\beta)}\right)$ is not bounded for $\beta<\alpha$, then also the sequence $\left(T_{n}\right)$ is not bounded for $\beta<\alpha$. Consequently for the validity of condition (3.2) it is necessary that $\beta \geq \alpha$. As $\alpha, \beta>-1$, then $C^{\beta}$ includes $C^{\alpha}$.

\section{References}

[1] A. Aasma. Transformations of summability fields. Acta et Comment. Univ. Tart., 770:38-51, 1987. 
[2] I. Albayrak and B.E. Rhoades. The question of equivalence for generalized Hausdorff matrices. J. Math. Anal. Appl., 328:414-428, 2007.

[3] L. Alpár. On the linear transformations of series summable in the sense of Cesàro. Acta Math. Hungar., 39:233-243, 1982.

[4] J. Baron. Introduction to the Theory of Summability of Series. Valgus, Tallinn, 1977.

[5] J. Boos. Classical and Modern Methods in Summability. Oxford University Press, Oxford, 2000.

[6] E. Savas and B. E. Rhoades. An inclusion theorem for double Cesàro matrices over the space of absolutely $k$-convergent double series. Appl. Math. Lett., 22:1462-1466, 2009.

[7] U. Stadtmüller and A. Tali. On some families of certain Nörlund methods and power series methods. J. Math. Anal. Appl., 238:44-66, 1999.

[8] U. Stadtmüller and A. Tali. Comparison of certain summability methods by speeds of convergence. Anal. Math., 29:227-242, 2003.

[9] U. Stadtmüller and A. Tali. A family of generalized Nörlund methods and related power series methods applied to double sequences. Math. Nachr., 282:288-306, 2009.

[10] B. Thorpe. Matrix transformations of Cesàro summable series. Acta Math. Hungar., 48:255-265, 1986. 